\title{
Manipulations of microfluidic droplets using electrorheological carrier fluid
}

\author{
Mengying Zhang, Jinbo Wu, Xize Niu, Weijia Wen, * and Ping Sheng \\ Department of Physics and Institute of Nano Science and Technology, The Hong Kong University of Science and Technology, \\ Clear Water Bay, Kowloon, Hong Kong
}

(Received 12 August 2008; published 9 December 2008)

\begin{abstract}
Electrorheological (ER) fluids are a type of "smart" colloid capable of reversible viscosity variations, or even solidification, in response to an applied electric field. The response time can be as short as a few milliseconds. By using the ER fluid as the carrier fluid in microfluidic chips, we report the generation and manipulation of microdroplets and bubbles via integrated, digitally controlled micro-electrodes equipped with a feedback system. By utilizing the strong electric response of the ER fluid, the flow rate can be easily controlled digitally, thereby making tunable the size of the droplets generated and their separations. In particular, ordering change in a chain of droplets is demonstrated. The maneuverability presented in this paper may have potential applications in a variety of lab chips for chemical reactions, bioassays, as well as microfluidic logic computation.
\end{abstract}

DOI: 10.1103/PhysRevE.78.066305

PACS number(s): 47.55.D-, 83.80.Gv

\section{INTRODUCTION}

Droplet-based microfluidics has the ability to perform a large number of reactions without increasing the device size or complexity [1-3]. In the form of droplets, reagents are conveyed precisely in discrete volumes, ranging from nanoliter to picoliter size. In the form of droplets, high throughput chemical reaction and single cell manipulation in biotesting can be achieved $[2,4]$. Furthermore, mixing of reagents in droplets has been proved to be achievable in millisecond(s), thus enabling multistep chemical reactions via droplet microfluids [5].

Droplet-based microfluidics involves the generation, detection and manipulation of discrete droplets inside micro devices $[6,7]$. Droplet generation or manipulation has generally been based on microfluidic channel geometry, such as flow-focusing geometry [8], as well as other active control method such as electrowetting on dielectric (EWOD) [9]. In particular, dielectric electrostatic forces have been employed to deflect droplets in microfluidic channels $[10,11]$. However, these forces are usually small compared to the hydrodynamic force in the microchannels; hence it is difficult to significantly alter droplets' flow behavior. While individual droplet manipulation is a highly desirable goal, its realization still remains a challenge [12].

Here we report a methodology to control liquid droplets and air bubbles via smart colliod, i.e., electrorheological (ER) fluid. Electrorheological fluids or suspensions constitute a type of colloid whose rheological characteristics are tunable under the application of an electric field. Under a sufficiently strong electric field, ER fluid can transform into an anisotropic solid, with a yield stress characterizing its strength. These rheological variations can occur within $10 \mathrm{~ms}$ and are reversible. Since the electric field can be generated through sensors, the rheological changes may be made in response to external environmental variations, hence the denotation of "smart" fluid. Recently, we have reported the

\footnotetext{
*phwen@ust.hk
}

applications of ER fluid in microfluidic chip, such as micropump [13], valve [14], and mixer [15]. In this work, we employ ER fluid as a carrier fluid to manipulate droplets and air bubbles.

\section{MATERIALS AND MICROFLUIDIC CHIP FABRICATION}

\section{A. Giant electrorheological (GER) fluid}

Our GER fluid consists of nanoparticles suspended in sunflower oil. The nanoparticles have a $50 \mathrm{~nm}$ core of barium titanyl oxalate, coated by a $\sim 5 \mathrm{~nm}$ layer of urea $[16,17]$. Under an applied field of $>1 \mathrm{kV} / \mathrm{mm}$, the GER fluid exhibits solidlike behavior, i.e., ability to transmit shear stress. The transformation from liquidlike to solidlike behavior is relatively fast, on the order of $1-10 \mathrm{~ms}$, and reversible. Compared to the conventional ER fluids, the GER fluid has a much larger ER effect under the same field strength. This is a crucial aspect of the GER fluid in our present work, so that sufficient pressure drop can be generated under a moderate electric field for significantly slowing down or stopping the microfluidic channel flow [14].

\section{B. Microfluidic chip}

The microfluidic chip shown in Fig. 1 has eight pairs of parallel electrodes, fabricated by metallic particle-PDMSbased conducting composite. Details concerning the conducting composite's fabrication process can be found in Ref. [18]. The function of electrodes $1-4$, located at the inlet of GER fluid, is to control droplet generation with desired liquid, such as water, while electrodes 5,7 , and 8 are used to control GER fluid flow through the side channel and the loop's two branches. Electrode 6, near to the triple junction, is for droplet detection. Details concerning the capacitance detection scheme can be found in Ref. [19]. The cross session of the main channel is $100 \mu \mathrm{m}$ in depth and $200 \mu \mathrm{m}$ in width (for the order-exchange loop, the width of channel is $300 \mu \mathrm{m})$ and its downstream is divided into three branch channels with one used as a by-pass channel while the other 


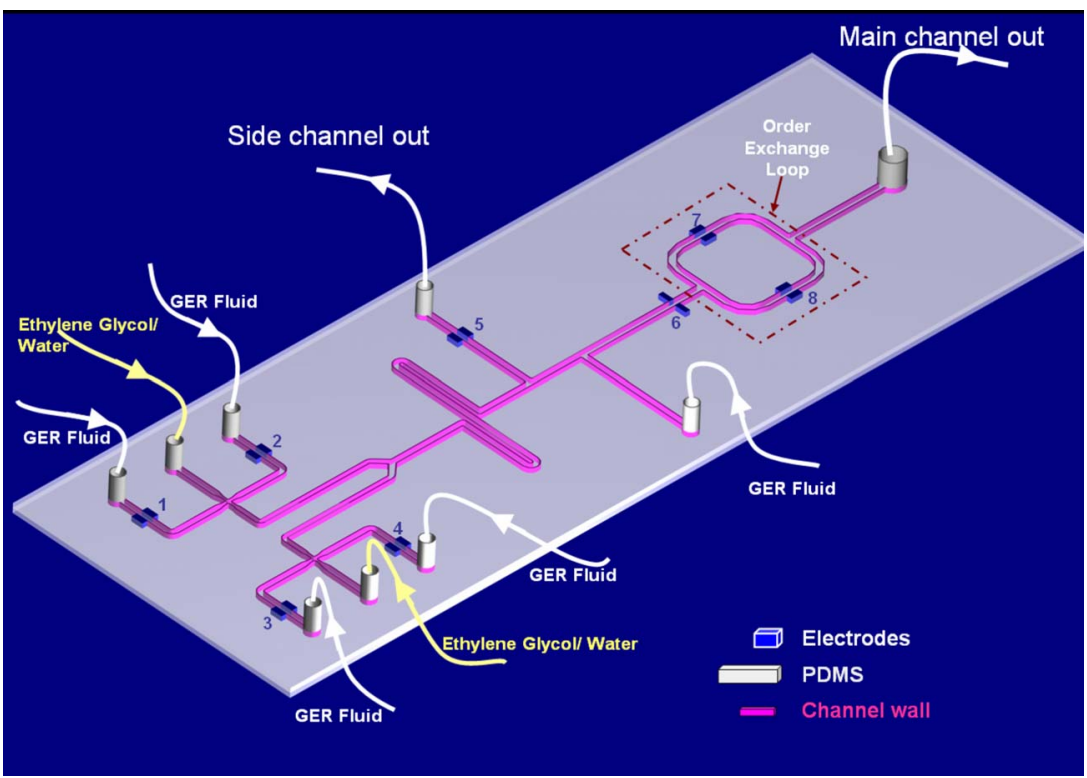

FIG. 1. (Color online) Schematic illustration of the microfluidic chip. The overall chip size is $3 \mathrm{~cm} \times 1.5 \mathrm{~cm} \times 0.4 \mathrm{~cm}$. The cross session of the main channel is $200 \mu \mathrm{m}$ in width and $100 \mu \mathrm{m}$ in height.

two are for droplet order exchange. The latter two combine into one again to form a loop with an outlet.

Soft lithographic technique was employed for the microchip fabrication. A negative photoresist SU8 was used to fabricate the channel mold. PDMS electrodes were first patterned with carbon-black-PDMS mixture. The mixture was placed on the substrate with the channel mold. After curing and bonding to another bottom layer of PDMS and embedding parallel electrodes on the channel walls [20], the microfluidic chip was completed. In the experiment, the chip was mounted on a platform and its electrodes were connected to a dc high voltage controller, with voltage adjustable in the range of 0 to $1500 \mathrm{~V}$ and frequency adjustable from 0 to $250 \mathrm{~Hz}$. Syringe pumps (KDS 200 syringes infusion pump) were used to inject the fluids into the chip through flexible tubes.

\section{EXPERIMENTAL RESULTS AND DISCUSSIONS}

\section{A. Generation of droplets}

Active control of the droplet generation process is very challenging, especially the variation of individual droplet size as a function of time. The current methord may achieve digitally controlled droplet generation on demand.

Liquid droplets may be generated by the flow-focusing approach or the T-junction approach as shown schematically in Figs. 2(a) and 2(b), respectively. In the flow-focusing approach (the one employed in Fig. 1), in which two streams of continuous-phase GER fluid (nanoparticles suspended in sunflower oil) were injected into the main channel through two side channels, and one stream of water, oil, or gas (the droplet fluid should be immiscible with sunflower oil, here we use water as an example) was injected via a center inlet. By properly controlling the flow rates of both the GER fluid and water, water droplets with targeted sizes can be generated. The control sequence is as follows. The GER fluid flow rate can be decreased by applying a voltage to the electrodes placing on the GER fluid inlets (electrodes 1-2 or 3-4 in Fig.
1), owing to the increased viscosity of the GER fluid. When the applied field is greater than $2 \mathrm{kV} / \mathrm{mm}$ (with applied voltage about $100 \mathrm{~V}$ when the channel width is about $200 \mu \mathrm{m}$ and in our experiment, we used $300 \mathrm{~V}$ to make sure the $E$ field is strong enough), the GER fluid stream can be tempo-

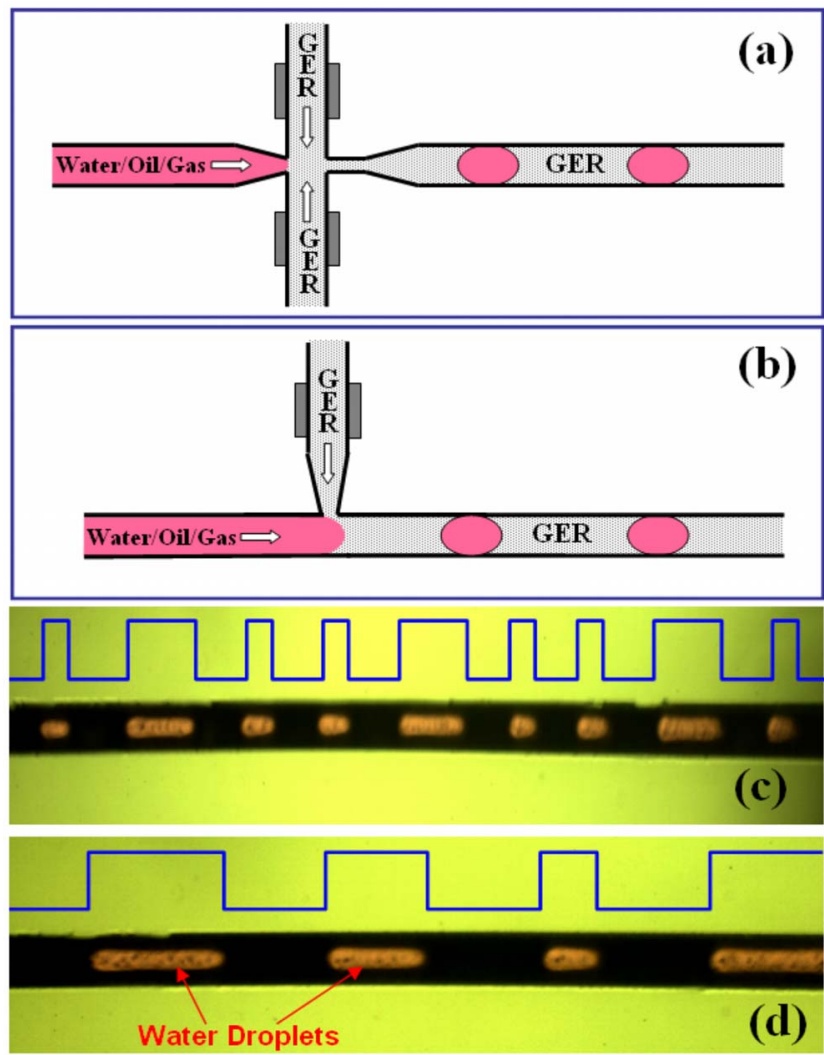

FIG. 2. (Color online) A schematic view of droplet generation: (a) flow-focusing approach; (b) $\mathrm{T}$ junction. (c) and (d) show the electric field controlled generation of droplets in a microchannel. Blue lines indicate the electric control signal applied to the electrodes embedded on two sides of the GER fluid inlets. 

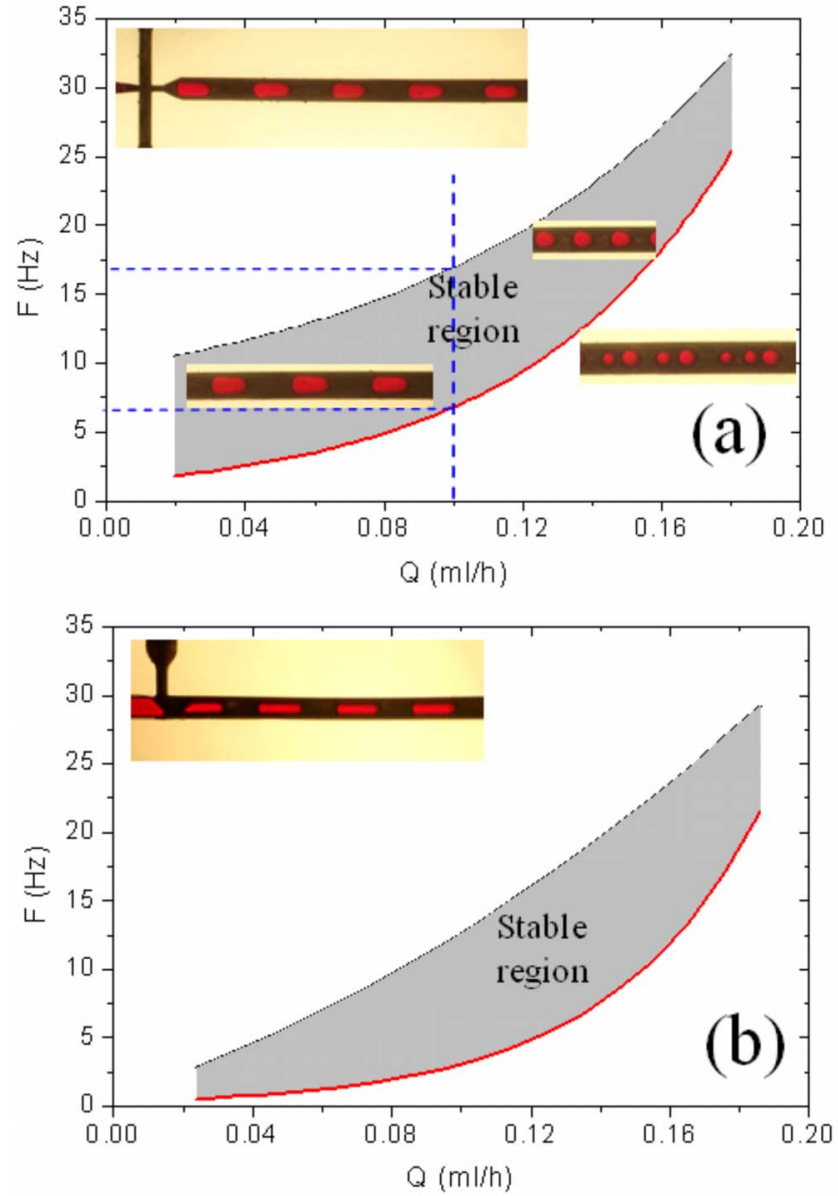

FIG. 3. (Color online) Frequency of droplet generation under the two different structures, plotted as a function of the flow rate. Insets are images of droplet generation under different conditions. The black dashed lines indicate the highest frequencies that stable droplet generation could be achieved. The red solid lines delineate the lowest frequencies. The shadow area between them is the stable droplet generation regime, where there is a one-to-one correspondence between the frequency of the applied electrical signal and the rate of droplet generation.

rarily stopped, with only water being injected into the main channel through the center inlet. When the electric field is decreased below the critical value or turned off, the GER fluid flow would rush into the main channel and temporarily stop the water stream. Thus the water droplets' generation can be electrically controlled. Similarly, in a T junction with one stream of water flowing into the main channel and another stream of GER fluid joining the water stream vertically at the junction, with the proper adjustment of the relative flow rates between the GER fluid and the water stream, water droplets could be formed. In both methods, the droplet size, and separation distance between two successive droplets may be tuned by adjusting the frequency and duty cycle of the control signals. Such control is illustrated in Figs. 2(c) and 2(d), in which the correspondence is shown between the electrical control signals applied to the electrodes (the blue line) and the profiles of generated water droplets.

We have also investigated the effect of frequency (of applied signals) at different injection rates. The results are
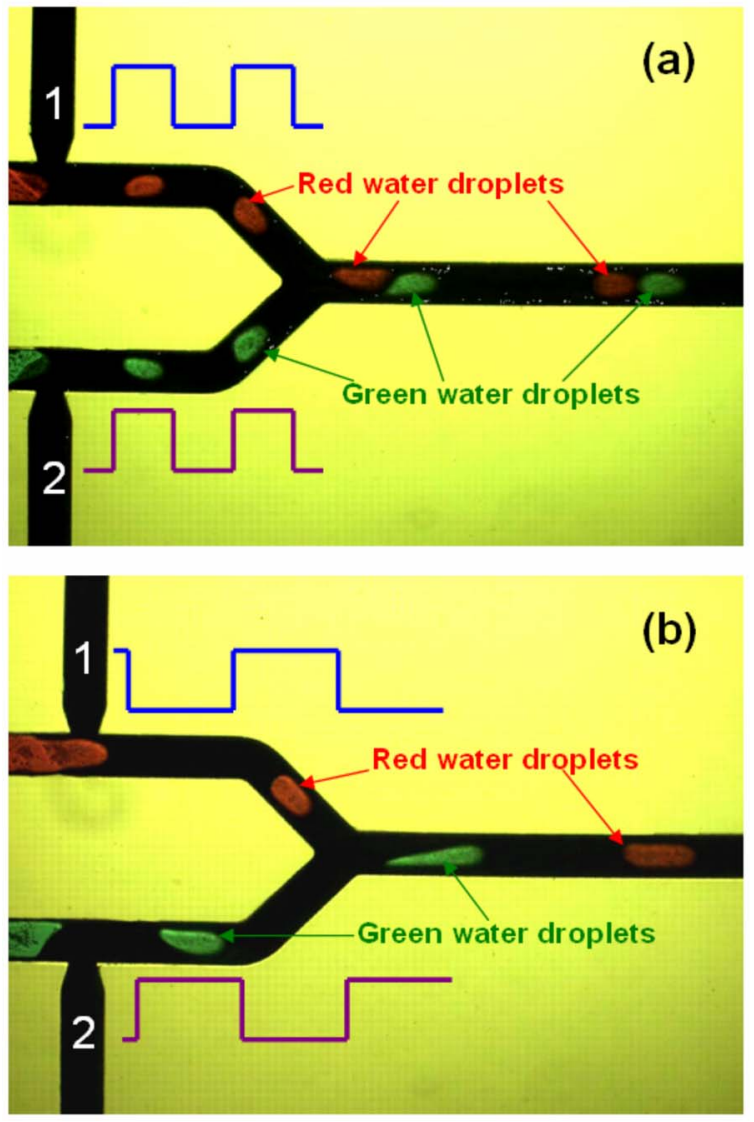

FIG. 4. (Color online) Controlled generation of two types of water droplets: (a) with the same phase of the control signals and (b) with opposite phase of the control signals. The blue and purple solid lines represent the control electric signals applied to the electrodes located next to the channels 1 and 2 .

shown in Fig. 3. Both the flow-focusing and T-junction approaches to droplet generation were investigated. The left upper insets of Figs. 3(a) and 3(b) are optical images of droplet profiles generated with the flow-focusing and T-junction structures, respectively, both carried out at a fixed flow rate without the electrical control signals. It should be noted that, without the application of control signals (no voltage applied to the GER fluid channel electrodes) the water droplet size was determined by the relative flow rates between the GER fluid stream and the water stream. This is denoted the passive approach to droplets generation. On the basis of passive droplet generation, water droplets with tunable sizes may be obtained by applying an electrical signal to the GER fluid stream. As a function of applied electrical signals' frequency, a stable generation region was found within which water droplet generation may be actively tuned by varying the frequency of the applied $E$ field. Within the stable regime, there is a one-to-one correspondence between the frequency of the applied control signal and the frequency or rate of droplet generation. That means the droplet generation frequency exactly equal to the frequency of the applied control signal. In Fig. 3 the stable droplet generation regime is delineated by the gray area in the frequency-flow rate map. For the flow-focusing structure if the flow rates of water and GER fluid were both fixed at $0.1 \mathrm{ml} / \mathrm{h}$, the generation rate of 


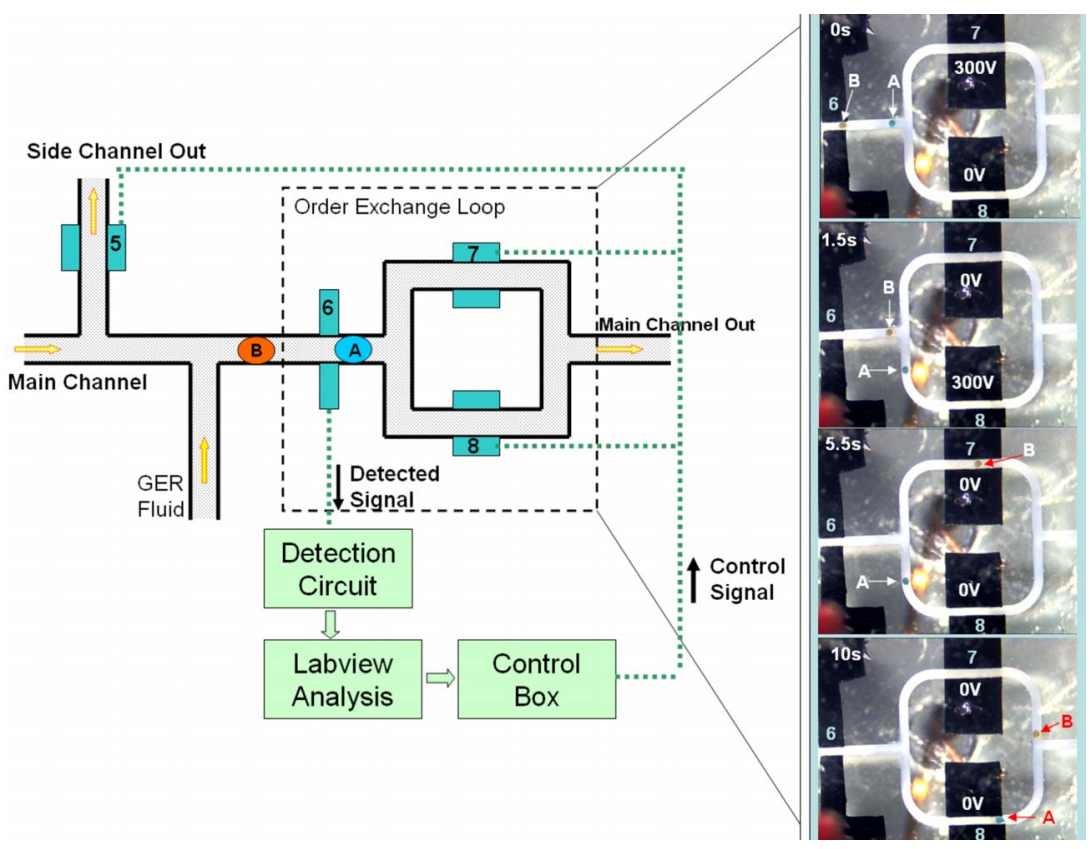

FIG. 5. (Color online) Flow chart and control circuit for droplet order exchange. The right insets are optical images taken at different times during the exchange process. droplet could be varied from 7 to 17 droplets/s (Hz) indicated by a dash line shown in Fig. 3(a). From the optical images, one can observe that in the stable region, uniform droplets train could be generated, and beyond the stable regime the droplet generation becomes unstable, characterized by irregularly spaced droplets of different sizes. From Figs. 3(a) and 3(b), we conclude that for both flow-focusing and T-junction structures the droplet generation rate and the droplet size can be easily tuned by external electric signals. We denote this the active droplet generation approach.

By using the T-junction approach, two types of droplets can be generated simultaneously to flow in the main channel, shown in Fig. 4. In Fig. 4(a), two types of droplets dyed red and green were generated with electrical pulse signals to electrodes 1 and 2 that were in phase. Uniform pairs of droplets are seen. In Fig. 4(b) we show the same two types of droplets generated with control signals to electrodes 1 and 2 that were out of phase with each other. In this case there is no pair formation.

\section{B. Droplets manipulation}

Once generated, the droplets can be manipulated relatively easily. Here we illustrate the use of flow rate control to switch the ordering(s) in a train of droplets. If one regards a train of different droplets as a coded message, then such switching implies the ability to revise or correct the message. The experimental setup and control mechanism are shown on the left panel of Fig. 5, where two droplets, the blue one (droplet A) and the orange one (droplet B) were generated from upstream and came into the main channel separated by a certain distance. Electrode 6, connected to the detection circuit, was used for droplet detection, achieved with a computer equipped with Labview software. When a droplet passes through electrode 6 , the detected signal triggers the control box to send the control signals to electrodes 5,7 , and 8 . The (on/off) control signals on these electrodes can thus be varied according to the requirements. As an example shown on the left in Fig. 5, one pair of droplets came from the upstream and passed through electrode 6 where the detected signals were collected and analyzed by detection circuit and Labview software. The control signals generated by the control box were sent to electrodes 5,7 , and 8 to instruct the $E$ field on electrode 5 be switched off so that other droplets coming from upstream would be pushed out of the side channel and only the two droplets were allowed into the order exchange loop. Another channel for the GER fluid was used to supply additional carrying fluid to continuously push the droplets into the exchange loop, as part of the GER fluid stream go by the way of the side channel (side channel was open at this moment). Once the two droplets enter the loop, the direction and relative residence time of the two droplets in the exchange loop can be controlled by the $E$-field status on electrodes 7 and 8 . As shown in the uppermost image the two droplets with a designated $A-B$ sequence approached the exchange loop. When a $300 \mathrm{~V}$ voltage was applied to electrode 7 while electrode 8 was off, the GER carrying fluid was stopped at the upper loop branch so that droplet $A$ went into the lower branch. Subsequently the voltage to electrode 8 was switched on while that on electrode 7 was switched off so that droplet $B$ went to the upper branch but droplet $A$ was stopped. By properly controlling the on-off duration of the applied voltages on electrodes 7 and 8 , droplets $A$ and $B$ resumed to move into the downstream main channel with the ordering reversed. Thus ordering and separation distance between the droplets can be adjusted in accordance to one's demand. Such maneuverability may be very useful in droplet control for biosystems and microfluidic computing.

\section{Bubble control}

By using a similar flow-focusing generation device as described above, the control of gas bubbles (such as $\mathrm{N}_{2}$ bubbles) was also demonstrated. Figures 6(a)-6(c) illustrate 


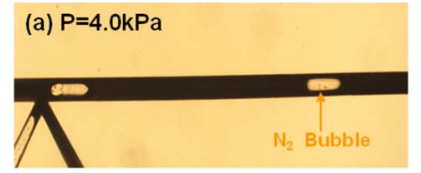

(b) $P=5.33 \mathrm{kPa}$

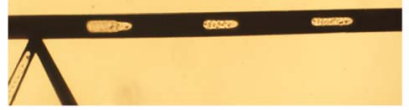

(c) $P=6.8 \mathrm{kPa}$

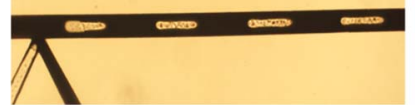

(d) $P=6.8 \mathrm{kPa}, T=400 \mathrm{~ms}$

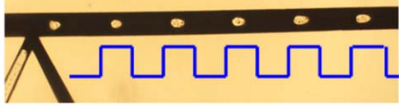

(e) $P=6.8 \mathrm{kPa}$, coding

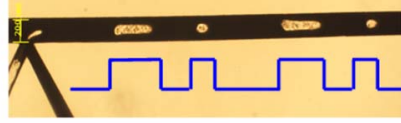

(f) $P=6.8 \mathrm{kPa}$, coding

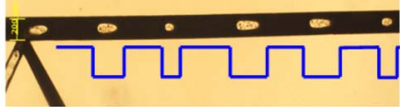

FIG. 6. (Color online) (a)-(c) $\mathrm{N}_{2}$ bubbles generated under different gas pressure but the same flow rate of the carrier fluid. No electrical control signal was applied. (e)-(g) Bubble generation under the same pressure and flow rate, but with different coded signals (indicated by the blue solid lines).

the generation of $\mathrm{N}_{2}$ bubbles, where different bubble sizes are shown to be produced by adjusting the inject pressure. Alternatively, since the GER fluid is electrically controllable, the bubble generation can be digitally controlled by applied electric signals without changing the gas pressure. This is shown in Figs. 6(d)-6(f), where different sizes of bubbles are shown to be generated digitally by adjusting the time duration of applied field to the GER fluid. Bubble generation control is an interesting topic especially in digital microfluidics [21], and it is also very useful for the application in biosystems. We note that the manipulation of bubbles is generally much more difficult, owing to their different characteristics compared with the liquid droplets. Nevertheless, the bubble size, flow direction as well as separation distance between the bubbles can be easily controlled with our approach.

\section{CONCLUDING REMARKS}

We report the implementation of droplet microfluidic chip using the GER fluid as the carrier fluid. It is shown that the generation and manipulation of droplets and gas bubbles may be achieved through digital signals applied to the embedded electrodes. In particular, the order exchange of the droplets was realized.

\section{ACKNOWLEDGMENTS}

The authors would like to acknowledge Hong Kong RGC Grant No. HKUST 621006 for the financial support of this project. The work was partially supported by the Nanoscience and Nanotechnology Program at HKUST
[1] B. Zheng and R. F. Ismagilov, Angew. Chem., Int. Ed. 44, 2520 (2005).

[2] S.-Y. Teh, R. Lin, L.-H. Hung, and A. P. Lee, Lab Chip 8, 198 (2008).

[3] D. M. Ratner, E. R. Murphy, M. Jhunjhunwala, D. A. Snyder, K. F. Jensen, and P. H. Seeberger, Chem. Commun. (Cambridge) 5, 578 (2005).

[4] H. Song, D. L. Chen, and R. F. Ismagilov, Angew. Chem., Int. Ed. 45, 7336 (2006).

[5] M. R. Bringer, C. J. Gerdts, H. Song, J. D. Tice, and R. F. Ismagilov, Philos. Trans. R. Soc. London, Ser. A 362, 1087 (2004).

[6] D. Belder, Angew. Chem., Int. Ed. 44, 3521 (2005).

[7] K. Jensen and A. P. Lee, Lab Chip 4, 31N (2004).

[8] S. L. Anna, N. Bontoux, and H. A. Stone, Appl. Phys. Lett. 82, 364 (2003).

[9] S. K. Cho, H. J. Moon, and C. J. Kim, J. Microelectromech. Syst. 12, 70 (2003).

[10] K. Ahn, C. Kerbage, T. P. Hunt, R. M. Westervelt, D. R. Link, and D. A. Weitz, Appl. Phys. Lett. 88, 024104 (2006).
[11] L. Wang, L. A. Flanagan, N. L. Jeon, E. Monuki, and A. P. Lee, Lab Chip 7, 1114 (2007).

[12] R. M. Lorenz, J. S. Edgar, G. D. M. Jeffries, and D. T. Chiu, Anal. Chem. 78, 6433 (2006).

[13] L. Liu, X. Chen, X. Niu, W. Wen, and P. Sheng, Appl. Phys. Lett. 89, 083505 (2006).

[14] L. Liu, X. Niu, W. Wen, and P. Sheng, Appl. Phys. Lett. 88, 173505 (2006)

[15] X. Z. Niu, L. Y. Liu, W. J. Wen, and P. Sheng, Phys. Rev. Lett. 97, 044501 (2006).

[16] W. Wen, X. Huang, S. Yang, K. Lu, and P. Sheng, Nature Mater. 2, 727 (2003).

[17] W. Wen, X. Huang, and P. Sheng, Soft Matter 4, 200 (2008).

[18] X. Niu, S. Peng, L. Liu, W. Wen, and P. Sheng, Adv. Mater. (Weinheim, Ger.) 19, 2682 (2007).

[19] X. Niu, M. Zhang, S. Peng, W. Wen, and P. Sheng, Biomicrofluidics 1, 044101 (2007).

[20] S. R. Quake and A. Scherer, Science 290, 1536 (2000).

[21] M. Prakash and N. Gershenfeld, Science 315, 832 (2007). 